PROCEEDINGS OF THE

AMERICAN MATHEMATICAL SOCIETY

Volume 131, Number 10, Pages 3021-3023

S 0002-9939(03)06954-5

Article electronically published on February 20, 2003

\title{
F-SPLIT GALOIS REPRESENTATIONS ARE POTENTIALLY ABELIAN
}

\author{
CHANDRASHEKHAR KHARE
}

(Communicated by David E. Rohrlich)

\begin{abstract}
In this note we relate the property of a semisimple $\ell$-adic Galois representation being " $F$-split" to its having abelian image.
\end{abstract}

If $E$ is an elliptic curve defined over a number field $K$, then it gives rise to a strictly compatible system $\left(\rho_{\ell}\right)$ of 2 -dimensional $\ell$-adic representations (see I-11 of [Se]) of the absolute Galois group $G_{K}$ of $K$. This arises from the action of $G_{K}$ on the $\ell$-adic Tate module $\mathrm{Ta}_{\ell}(E)$ of $E$.

This strictly compatible system has very different properties when $E$ has CM and when $E$ does not have CM. For instance in the latter case the image of $\rho_{\ell}$ is all of $G L_{2}\left(\mathbf{Z}_{\ell}\right)$ for almost all $\ell$ while in the former case $\left(\rho_{\ell}\right)$ is potentially abelian, i.e., $\left.\rho_{\ell}\right|_{G_{L}}$ is abelian for all $\ell$ for a fixed finite extension $L$ of $K$. These cases are also markedly different from the point of view of the the distribution of the eigenvalues of the Frobenii (see I-25 of [Se]).

Yet another notable difference is in the behaviour of the compositum of the splitting fields of the Frobenius polynomials attached to $E$, i.e., characteristic polynomials of $\left.\rho_{\ell}\right|_{G_{L}}\left(\mathrm{Frob}_{r}\right)$ ( $r$ prime to $\ell$ ) for primes $r$ of $L$ at which $E_{/ L}$ has good reduction: these characteristic polynomials depend only on $r$ and not on $\ell$, and here again $L$ is a fixed number field which contains $K$. When $E$ does not have $\mathrm{CM}$, this compositum is always an infinite extension of $\mathbf{Q}$ (see exercise on page IV-13 of [Se]). If $E$ has CM, this compositum is a finite extension whenever all the endomorphisms (i.e., CM) of $E$ are defined over $L$.

This difference is the most pertinent to this short note. We study here in a more abstract setting the relationship between a compatible system of $\ell$-adic Galois representations being potentially abelian and the nature of the field generated by the splitting fields of the Frobenius polynomials.

Definition 1. Let $E, F$ be number fields and fix embeddings of $E, F$ in each completion of $\overline{\mathbf{Q}}$.

(1) Consider $\left(\rho_{\lambda}\right)$ a strictly compatible system of $E$-rational, continuous, semisimple, $n$-dimensional, $\lambda$-adic representations $\rho_{\lambda}: G_{K} \rightarrow G L_{n}\left(E_{\lambda}\right)$ for a number field $K$ and $\lambda$ running through the places of $E$ (see I-11 and I-13 of [Se]). We say that $\left(\rho_{\lambda}\right)$ is $F$-split if for almost all places $r$ of $L$ the characteristic polynomial of $\rho_{\lambda}\left(\right.$ Frob $\left._{r}\right)$ (which is defined when $r$ and $\lambda$ are of coprime residue characteristics, and then is independent of $\lambda$ ) splits over

Received by the editors May 13, 2002.

2000 Mathematics Subject Classification. Primary 11R32. 
$F$. We say that $\left(\rho_{\lambda}\right)$ is potentially $F$-split if $\left(\left.\rho_{\lambda}\right|_{G_{L}}\right)$ is $F$-split for a finite extension $L$ of $K$.

(2) We say that a continuous, semisimple representation $\rho_{\lambda}: G_{K} \rightarrow G L_{n}\left(E_{\lambda}\right)$ is $F$-split if the characteristic polynomial of $\rho_{\lambda}\left(\operatorname{Frob}_{r}\right)$ of $L$ for all places $r$ of $L$ unramified in $\rho_{\lambda}$ splits over $F$. We say that $\rho_{\lambda}$ is potentially $F$-split if $\left.\rho_{\lambda}\right|_{G_{L}}$ is $F$-split for a finite extension $L$ of $K$. (Note: By [KhRa], the set of places $r$ of $L$ unramified in $\rho_{\lambda}$ has density 1.)

Definition 2. We say that either a strictly compatible system $\left(\rho_{\lambda}\right)$ of $E$-rational, continuous, semisimple, $n$-dimensional $\lambda$-adic representations of $G_{K}$ or an $E$ rational, continuous, semisimple representation $\rho_{\lambda}: G_{K} \rightarrow G L_{n}\left(E_{\lambda}\right)$ is abelian (resp., potentially abelian) if each $\rho_{\lambda}$ has abelian image (resp., if for some finite extension $L$ of $K$ each $\left.\rho_{\lambda}\right|_{G_{L}}$ has abelian image).

Theorem 1. A strictly compatible system $\left(\rho_{\lambda}\right)$ of E-rational, continuous, semisimple, $n$-dimensional $\lambda$-adic representations of $G_{K}$ is potentially $F$-split for some number field $F$ if and only if it is potentially abelian.

Proof. We first prove the "only if" statement of the theorem. By restriction of scalars, it is enough to prove that a Q-rational, continuous, semisimple, $n$ dimensional $\ell$-adic system of representations of $G_{K}$ that is potentially $F$-split for some number field $F$ is potentially abelian. (We reduce to this case for ease of comparison with $\left[\overline{\mathrm{LP}}\right.$.) Consider a prime $\ell_{0}$ that splits completely in $F$. Let $L$ be a finite extension of $K$ such that the compatible system $\left(\left.\rho_{\ell}\right|_{G_{L}}\right)$ is $F$-split and such that the Zariski closure of the image $\rho_{\ell_{0}}\left(G_{L}\right)$ is connected (such an $L$ exists!). From the assumption of $F$-splitness and the Cebotarev density theorem we deduce that the subgroup $\rho_{\ell_{0}}\left(G_{L}\right)$ of $G L_{n}\left(\mathbf{Q}_{\ell_{0}}\right)$ contains no non-split torus. Since $\rho_{\ell}$ is semisimple this implies that the $\ell_{0}$-adic representation has toral image (and in particular is abelian). This together with the $\mathbf{Q}$-rationality of $\rho_{\ell_{0}}$, and a consequence of a result of Waldschmidt in transcendental number theory (see Theorem 2 of $[\mathrm{H}]$ ), implies that $\rho_{\ell_{0}}$ arises as the direct sum of 1-dimensional representations arising from algebraic Hecke characters $\chi_{i}$ of $L, i=1, \cdots, n$. The $\chi_{i}$ 's give rise (see II of $[\mathrm{H}]$ ) to a strictly compatible system of (continuous, semisimple, $n$-dimensional) $\lambda$-adic representations. Comparing this with $\left(\left.\rho_{\ell}\right|_{G_{L}}\right)$, we deduce that $\left(\left.\rho_{\ell}\right|_{G_{L}}\right)$ itself "arises" from the direct sum of the algebraic Hecke characters $\chi_{i}$ proving the proposition. (Note that without using $[\mathrm{H}]$ it follows from Proposition 6.14 of $[\mathrm{LP}]$ that there is a finite extension $L$ of $K$ such that for a density 1 set of primes $\ell$, $\left.\rho_{\ell}\right|_{G_{L}}$ has abelian image, and thus $\left(\left.\rho_{\ell}\right|_{G_{L}}\right)$ is an "abelian system" with $\ell$ running through a density 1 set of primes. But we do not get the stronger assertion that $\left(\left.\rho_{\ell}\right|_{G_{L}}\right)$ itself is an abelian system.)

We now prove the other direction of the statement of the theorem. Consider a strictly compatible system $\left(\rho_{\lambda}\right)$ that is potentially abelian and consider an extension $L$ of $K$ such that $\left(\left.\rho_{\lambda}\right|_{G_{L}}\right)$ is abelian. Choose any place $\lambda$ of $E$. By appealing to [H] again, and using that $\rho_{\lambda}$ is rational over $E$, semisimple and abelian, we deduce that $\left.\rho_{\lambda}\right|_{G_{L}}$ arises as the direct sum of 1-dimensional representations arising from algebraic Hecke characters $\chi_{i}$ of $L$. Then by standard proprties of algebraic Hecke characters (see II of $[\overline{\mathrm{H}}]$ ), we conclude that $\left.\rho_{\lambda}\right|_{G_{L}}$ is $F$-split for some number field $F$, which in its turn implies that $\left(\left.\rho_{\lambda}\right|_{G_{L}}\right)$ is an $F$-split strictly compatible system.

Remark. While it is true that an $E$-rational abelian, semisimple representation $\rho_{\lambda}$ (or compatible system $\left(\rho_{\lambda}\right)$ ) is always $F$-split for some number field $F$ (as follows 
from the proof), it is not true that an $F$-split representation $\rho_{\lambda}$ (or compatible system $\left(\rho_{\lambda}\right)$ ) is abelian (consider the "constant", compatible systems arising from Artin representations).

The case of a single, $F$-split, $\lambda$-adic representation of $G_{K}$ we cannot settle, even in the case when the image is in $G L_{2}\left(\mathbf{Q}_{\ell}\right)$ (if the completions of $F$ contain all the quadratic extensions of $\mathbf{Q}_{\ell}$ we do not know how to proceed). We end with a question.

Question 1. Is a continuous, semisimple, $F$-split $\lambda$-adic representation $\rho: G_{K} \rightarrow$ $G L_{n}\left(E_{\lambda}\right)$ potentially abelian?

\section{ACKNOWLEDGEMENTS}

I thank Richard Pink, Dipendra Prasad and J-P. Serre for helpful correspondence: each came up with different proofs of the theorem of this note (independently of each other and of the author, when responding to messages posing mainly the question above). The terminology " $F$-split" was suggested by Richard Pink, and J-P. Serre pointed out the example in his book. I also thank David Rohrlich for helpful comments that improved the exposition.

\section{REFERENCES}

[H] Henniart, G., Représentations $\ell$-adiques abéliennes, in Séminaire de Théorie des Nombres, Progress in Math. 22 (1982), 107-126, Birkhauser. MR 85d:11070

[KhRa] Khare, C., Rajan, C. S., The density of ramified primes in semisimple p-adic Galois representations, International Mathematics Research Notices no. 12 (2001), 601-607. MR 2002e:11066

[LP] Larsen, M., Pink, R., On $\ell$-independence of algebraic monodromy groups in compatible systems of representations, Invent. Math. 107 (1992), 603-636. MR 93h:22031

[Se] Serre, J-P., Abelian $\ell$-adic representations and elliptic curves, Addison-Wesley, 1989. MR 91b:11071

Department of Mathematics, University of Utah, 155 S 1400 E, Salt lake City, Utah 84112

E-mail address: shekhar@math.utah.edu

Current address: School of Mathematics, TIFR, Homi Bhabha Road, Mumbai 400 005, India

E-mail address: shekhar@math.tifr.res.in 